\title{
Computer Simulation of Ion Beam Analysis of Laterally Inhomogeneous Materials
}

\author{
M. Mayer \\ Max-Planck-Institut für Plasmaphysik, Boltzmannstr. 2, D-85748 Garching, \\ Germany
}

\begin{abstract}
The program STRUCTNRA for the simulation of ion beam analysis charged particle spectra from arbitrary two-dimensional distributions of materials is described. The code is validated by comparison to experimental backscattering data from a silicon grating on tantalum at different orientations and incident angles. Simulated spectra for several types of rough thin layers and a chessboard-like arrangement of materials as example for a multi-phase agglomerate material are presented. Ambiguities between back-scattering spectra from two-dimensional and one-dimensional sample structures are discussed.
\end{abstract}

\section{Introduction}

Ion beam analysis (IBA) methods such as Rutherford back-scattering (RBS), elastic recoil detection analysis (ERDA), nuclear reaction analysis (NRA) and medium energy ion scattering (MEIS) are considered as quantitative methods for depth profiling of elements in the near-surface layer of solids [1]. In a strict sense, however, this is only true for one-dimensional laterally homogeneous materials, i.e. where the material distribution parallel to the surface is homogeneous. In this case concentrations of elements can be described as function of depth by depth profiles. Popular simulation codes for IBA [2] such as SIMNRA [3,4] or NDF [5] describe depth profiles as layered structures in slab geometry assuming atomic mixing of the constituents within each layer.

The success of IBA methods in analyzing one-dimensional sample structures widened the application of IBA methods to analysis of laterally inhomogeneous samples using the same well-developed methods as for one-dimensional samples [6]. Laterally inhomogeneous samples are two- or three-dimensional structures and include all types of rough layers and surfaces; porous materials; or compound multi-phase materials like geological samples, sinter materials, 
paint, or collections of dust particles. These compound multi-phase materials without layered structure will be called heterogeneous agglomerate materials throughout this paper.

Several models with different levels of generality have been developed for the simulation of IBA spectra from rough substrates or rough layers [7-20] using analytical approximations, straight line models (where incident and exit paths are approximated as straight lines) or Monte-Carlo simulations. Porosity with random distribution of pores can be treated as additional energy spread contribution if the diameter of pores is sufficiently small [21].

The simulation of MEIS spectra from three-dimensional nano-structures at the surface of a substrate has been implemented in the program PowerMeis [22]. The code RBS-MAST [23] allows to simulate RBS spectra from twoor three-dimensional sample structures but neglects all energy spread contributions (energy-loss straggling, multiple scattering, detector resolution etc.). The Monte-Carlo code CORTEO [24] has been recently extended to use twoor three-dimensional sample structures as input [25].

This paper describes the STRUCTNRA program which allows simulation of two-dimensional sample structures using the well-known SIMNRA program as simulation kernel. The program is validated by comparison to experimental backscattering spectra from a silicon grating structure. Examples for spectra from various rough layers and two-dimensional arrangements of elements are given, and ambiguities between spectra obtained from one- and two-dimensional sample structures are discussed.

\section{Computer simulation}

\subsection{Code structure}

The simulation code STRUCTNRA allows the simulation of RBS, ERDA, NRA and MEIS spectra from arbitrary two-dimensional structures. The structure can be an image acquired by scanning electron (SEM) or transmission electron microscopy (TEM), or an arbitrary drawing, see Fig. 1. STRUCTNRA allows import of images in 24-bit bitmap (BMP) format. Each color in the image represents a material. A pixel can be empty, or it can contain a material consisting of up to 40 different elements and a mass density. STRUCTNRA uses quadratic pixels with adjustable pixel size $\Delta$, see Fig. 1. Periodic boundary conditions are applied in horizontal direction.

STRUCTNRA uses SIMNRA 6.93 or higher [3,4] as simulation kernel through 
its COM interfaces. All SIMNRA features are available for simulations: NonRutherford and NRA cross-sections, several stopping power data sets, correction factors to Bragg's rule for individual materials, electronic energy loss straggling, finite detector resolution, energy spread by multiple small angle scattering, filter foils in front of the detector, etc. Simulations in back- and forward-scattering and in transmission geometry are possible.

STRUCTNRA assumes trajectories of incident and outgoing particles to be straight, see Fig. 1. Incident trajectories (solid line in Fig. 1) start at quasirandom starting points at the upper edge of the image. Quasi-random starting points are selected due to their more homogeneous coverage of the horizontal direction than a random distribution of starting points. The intersection points between the incident trajectory and the pixel grid are calculated and the path within each pixel is used as layer. This sequence of layers defines the target for incident particles $T_{i n}$, see the dash-dotted lines in Fig. 1 for the layer boundaries of the first 3 layers of $T_{i n}$. The sub-spectrum from each individual pixel (more precisely: from the incident trajectory inside of each pixel) is calculated by constructing a target $T_{\text {out }}$ for outgoing particles by starting at the middle point of the path inside the pixel and calculating the intersection points between the outgoing trajectory and the pixel grid, see the dashed line in Fig. 1. This sequence defines the layer structure for the target for outgoing particles $T_{\text {out }} . T_{\text {in }}$ and $T_{\text {out }}$ are connected at the upper edge of the currently calculated pixel: This is realized by an additional shift of $T_{\text {out }}$ versus $T_{i n}$. Then the next pixel on the incident trajectory is calculated in the same way by constructing a new target $T_{\text {out }}$ (dotted line in Fig. 1). This is repeated until the energy of incident particles decreases below a preset threshold energy typically close to zero.

Multiple small-angle scattering is taken into account as energy spread, lateral spread is neglected. Dual large-angle scattering [26] is approximated in slab geometry, with the composition of each slab as average composition of all pixels in a row.

The necessary pixel size depends on incident particle species, energy, and materials of the pixels. Typical pixel sizes are in the range from a few nm to a few ten $\mathrm{nm}$. Too large pixels can result in distortions of simulated spectra, too small pixels increase the computing time. The number of necessary incident trajectories depends on the complexity of the structure, typically between hundred and a few thousand trajectories are necessary. Typically a few thousand to a few millions of SIMNRA spectra have to be calculated. The computing time is proportional to the number of incident trajectories, the computing time for each trajectory is between $\propto m$ and $\propto m^{2}$ (with $m$ the number of traversed pixels in vertical direction).

STRUCTNRA uses 2-dimensional sample structures, i.e. the sample shown in 
Fig. 1 is assumed to extend infinitely perpendicular to the paper plane. The plane of the sample cross-section is identical to the plane spawned by incident and exit beams. Note that at some geometries (for example Cornell geometry at oblique incidence) the cross-section of this plane with the sample may be non-parallel to the surface normal.

The spectrum of a random 3-dimensional sample, such as a rough surface without preferential lateral orientation or a porous layer without texture, is identical to the spectrum calculated from the 2-dimensional section of the sample with the plane spawned by incident and exit beams. The spectrum of a regular 3-dimensional object or structure (for example a spherical or cylindrical structure at the surface, see [22, Fig. 2] for illustration; or a 3-dimensional heterostructure) can be calculated as linear superpositions of spectra of 2dimensional sample sections at different z-positions, where the z-axis is perpendicular to the paper plane in Fig. 1.

\subsection{Comparison to experimental data}

For code validation experimental RBS data were measured from a two-dimensional silicon grating on tantalum with $1500 \mathrm{keV}$ incident ${ }^{4} \mathrm{He}$ ions at a scattering angle of $165^{\circ}$. The measurements were performed in parallel geometry where the detection plane, i.e. the plane formed by incident and exit beams, is parallel to the grating structure (see Fig. 2a), and in perpendicular geometry at different incident angles with the detection plane perpendicular to the grating structure (see Fig. 2b). A focused ion beam (FIB) cross-section of the grating structure is shown in Fig. 2c). Based on the quantitative evaluation of several FIB cross-sections and by fitting RBS spectra measured at $1000 \mathrm{keV}$ (not shown here) and $1500 \mathrm{keV}$ at normal incidence, $45^{\circ}$ and $60^{\circ}$ incident angles an idealized grating structure was derived and is shown in Fig. 2d). This sample structure was used for all subsequent simulations. As can be seen from the FIB cross-section the side walls are not perfectly perpendicular but are slightly inclined. This inclination is difficult to measure quantitatively from the FIB images, but can be determined from the RBS spectra in parallel orientation as already described in [20]. Comparing Si layer thicknesses derived from FIB cross-sections and from RBS data yields the atomic density of the Si layer, which was natural density within the uncertainty of this measurement.

RBS spectra in parallel orientation at normal incidence and in perpendicular orientation at normal incidence, $45^{\circ}$ and $60^{\circ}$ incident angles are shown in Fig. 3 together with the corresponding simulations using the target structure from Fig. 2d). The signal from the silicon grating is in channels below 300, but overlaps with the backscattering signal from tantalum and is difficult to evaluate quantitatively. The high-energy edge of tantalum is shifted towards lower 
energies, this shift is influenced by the silicon layer thicknesses and the lateral distribution of silicon. With parallel sample orientation (see Fig. 2a)) incident and exit ions traverse identical silicon thicknesses. The spectrum therefore has two steps: One step at channels around 460 due to ions traversing the thin part of the Si layer on incident and exit paths and a second step at channels around 420 due to ions traversing the thick part of the Si layer. The inclination of the plateau in channels 430-450 depends on the inclination of the side walls as already described in [20]. The measured spectrum can be well reproduced in the simulation, see [20] for details.

The spectrum at perpendicular orientation and normal incidence is quite different from the spectrum at parallel orientation, see Fig. 3. As described above the step at channels around 460 is caused by particles with trajectories having incident and exit paths through the thin part of the silicon layer. Compared to the parallel case this step decreases by a factor of about 2 at perpendicular orientation due to the decreased probability that both incident and exit paths pass through the thin part of the Si layer and an increased probability of incident trajectory through the thin layer and exit trajectory through some fraction of the thick part of the Si layer. The second step at channels around 420 disappears almost completely due to the vanishing probability that ions pass the thick part of the layer both on their incident and exit paths: If an incident ion passes the thick layer on its incident path then the exit path is most probably either through the side wall or through the thin layer. The simulation fits the measured spectrum very well.

At oblique incidence the detection plane and the cross-sectional plane through the sample are tilted by a tilt angle $\alpha$. This can be taken into account in simulations by stretching the sample in vertical direction by a factor $1 / \cos \alpha$. Measured and simulated spectra for incident angles of $45^{\circ}$ and $60^{\circ}$ are shown in Fig. 3. The Ta edge is shifted towards lower energies due to the increasing silicon layer thickness and changes its shape due to increasing probability of trajectories with incident path through the thin Si layer and exit path through some fraction of the thick layer or incident path through the thick layer and exit through the side wall or the thin layer. At $45^{\circ}$ incidence the step due to both incident and exit paths through the thin layer has shifted to channels around 430 and further decreased in height, at $60^{\circ}$ incidence this step has shifted to channels around 380 and has almost disappeared. The simulation generally reproduces the shape of the spectra very well, but with some tendency to overestimate the contribution of the step around channel 430 at $45^{\circ}$ and the step around channel 380 at $60^{\circ}$. This may be due to the neglect of lateral spread caused by multiple small-angle scattering in the simulations, but other explanations (such as a small misalignment of the grating with respect to the plane formed by incident and exit beams) cannot be fully excluded. 


\section{Results}

\subsection{Rough layers}

A rough triangular gold layer on top of a graphite substrate is shown in Fig. 4a). The structure extends infinitely perpendicular to the plane shown in Fig. 4a). Both materials have natural densities of $19.3 \mathrm{~g} / \mathrm{cm}^{3}$ (Au) and $2.27 \mathrm{~g} / \mathrm{cm}^{3}(\mathrm{C})$. The mean thickness of the gold layer is $450 \mathrm{~nm}$ with a maximum thickness of $900 \mathrm{~nm}$, resulting in a total amount of gold of $2.66 \times$ $10^{18}$ atoms $/ \mathrm{cm}^{2}$.

The calculated back-scattering spectrum from this rough layer is shown in Fig. 4c) (solid line) for $2 \mathrm{MeV}^{4} \mathrm{He}$ ions at normal incidence and a scattering angle of $165^{\circ}$. The spectrum was calculated by STRUCTNRA using the SigmaCalc cross-section for back-scattering from carbon [27] and SRIM 2003 stopping powers [28]. The scattering geometry is indicated by the two arrows in Fig. 4a). A broad beam irradiating the whole structure is assumed. The pixel size was $2.5 \times 2.5 \mathrm{~nm}^{2}$.

Hypothetical depth profiles of gold and carbon are shown in Fig. 4b). The back-scattering spectrum from a sample having this depth profile was calculated by SIMNRA using the same geometry and input data as for the rough layer and is shown in Fig. 4c) as dashed line: The spectrum from the rough layer and the spectrum from the depth profile are practically indistinguishable. This example illustrates the ambiguity between surface roughness and depth profiles: Without further knowledge the spectrum from a rough layer can be easily misinterpreted as depth profile (and vice versa). This ambiguity is well known. One of the first descriptions can be found in [29, Appendix E], where it was shown that the back-scattering spectrum of a laterally nonuniform lead layer on silicon substrate could be misinterpreted as diffusion profile of lead into silicon. Another example was published in [30]. Nevertheless, this ambiguity between layer roughness and depth profile and the possible misinterpretation of roughness as depth profile is still a frequent misinterpretation of IBA spectra. One possible method to exclude this misinterpretation is scanning electron microscopy (SEM) of the sample surface for proving lateral homogeneity of the material distribution. Multiple measurements with different ion species / different energies / different incident angles / different geometries may indicate potential problems, but it remains subject of future research to show if multiple measurements are always able to distinguish between roughness and depth profile.

The depth profile shown in Fig. 4b) has little in common with the structure shown in Fig. 4a), except the qualitative statement that the gold is distributed 
somehow on top of the graphite. Nevertheless, one can ask the question if the depth profile in Fig. 4b) has some hidden truth in it. The total amount of gold extracted from the depth profile is $2.83 \times 10^{18}$ atoms $/ \mathrm{cm}^{2}$, which is at least close to the amount of gold in the rough layer of $2.66 \times 10^{18}$ atoms $/ \mathrm{cm}^{2}$.

A smooth gold layer and layers with different roughness are shown in Fig. 5a). The structures extend infinitely perpendicular to the plane shown in Fig. 5a), periodic boundary conditions are applied in horizontal direction. Simulated RBS spectra using STRUCTNRA are shown in Fig. 5b) for $2 \mathrm{MeV}^{4} \mathrm{He}$, normal incidence, scattering angle $165^{\circ}$. The gold layers were assumed to have natural density, SRIM 2003 stopping powers were used. The total number of gold atoms is identical for all layers. As can be seen from Fig. 5b) the shape of the spectra depends strongly on the lateral arrangement of gold in the layer. Nevertheless, for not too extreme roughnesses the count integrals of the spectra change only moderately, see Fig. 5c). Except for the cases 'Step 100/800 nm' and the 'Needle' structure the count integrals are within $10 \%$ of the smooth layer. I.e. for not too extreme roughness, not too large energy losses and sufficiently smooth cross-sections (such as Rutherford cross-sections) count integrals allow to determine total amounts of elements with an uncertainty of the order of $10 \%$. For smaller energy losses the uncertainty gets smaller. For many applications this accuracy is already sufficient.

The shape of the spectra shown in Fig. 5b) is determined by the path lengths inside the material on the incident paths, the exit paths, and by correlations between incident and exit paths. The total number of back-scattered particles, i.e. the count integral, however, depends only on the path length distributions on incident paths. For the smooth Au layer in Fig. 5a) the energy loss is $302.9 \mathrm{keV}$, the cross-section at the surface is $8.22 \mathrm{barn} / \mathrm{sr}$ and increases to $11.38 \mathrm{barn} / \mathrm{sr}$ at the rear side of the layer with a mean crosssection of $9.80 \mathrm{barn} / \mathrm{sr}$. For the 'Step 300/600 nm' example the energy losses are $200.0 \mathrm{keV}$ and $407.1 \mathrm{keV}$ in the two steps, resulting in mean cross-sections of $9.17 \mathrm{barn} / \mathrm{sr}$ and $10.56 \mathrm{barn} / \mathrm{sr}$ and an average cross-section of $9.87 \mathrm{barn} / \mathrm{sr}$. This is very close to the mean cross-section of the smooth layer and results in quite similar numbers of back-scattered particles.

Count integrals are therefore relatively robust numbers for determining total amounts of elements in rough thin layers, provided that no particles are lost (i.e. that all scattered particles reach the detector and are not lost inside the layer). Due to the same reason the approximation of a rough thin layer by a depth profile can give total amounts of elements close to the correct amount, despite the fact that the depth profile itself is meaningless. Nevertheless, in a particular case it is always advisable to measure the roughness profile and to simulate the spectra based on this roughness profile in order estimate possible errors. 


\subsection{Heterogeneous agglomerate materials}

A simple example of a heterogeneous agglomerate material is shown in Fig. 6a). The material consists of gold (white squares) and carbon (black squares) with chessboard square size $\Delta$, the structure extends infinitely perpendicular to the plane shown in Fig. 6a). For simplicity the atomic densities of $\mathrm{Au}$ and $\mathrm{C}$ are assumed to be identical with $8 \times 10^{22}$ atoms $/ \mathrm{cm}^{3}$ : In this case the volume fractions are identical to the atomic fractions and are $50 \% \mathrm{Au}$ and $50 \% \mathrm{C}$.

Simulated back-scattering spectra using STRUCTNRA for $2.5 \mathrm{MeV}$ protons at normal incidence and a scattering angle of $165^{\circ}$ are shown in Fig. 6b) for different chessboard square sizes. SRIM 2003 stopping powers and the SigmaCalc cross-section for C [27] were used. Dashed lines are SIMNRA results for infinitely large and indefinitely small squares. Indefinitely small squares correspond to atomic mixing, the spectrum then can be calculated from a layer with composition $\mathrm{Au}_{0.5} \mathrm{C}_{0.5}$. For infinitely large squares the spectrum is a linear superposition of 0.5 times the spectrum from bulk $\mathrm{Au}$ and 0.5 times the spectrum from bulk $\mathrm{C}$. The factors 0.5 are the fractions of the sample area covered by the corresponding material. For infinitely large squares correlations (such as incidence through an Au square and exit through a $\mathrm{C}$ square) can be neglected. The simulations for infinitely large and $100 \mu \mathrm{m}$ large squares agree very well, as do the simulations for indefinitely small and $0.1 \mu \mathrm{m}$ large squares.

The Au contributions to the spectra differ by a factor of about 2 between infinitely large and indefinitely small squares. Why is this the case? For indefinitely small squares, i.e. for atomic mixing of $\mathrm{Au}$ and $\mathrm{C}$, the height of the $\mathrm{Au}$ spectrum close to the surface is

$$
N_{A u} \propto \frac{c_{A u}}{c_{A u} S_{A u}^{e f f}+c_{C} S_{C}^{e f f}} \approx \frac{1}{S_{A u}^{e f f}}
$$

with $N_{A u}$ the number of counts per channel, $c_{A u}$ and $c_{C}$ the atomic fractions of $\mathrm{Au}$ and $\mathrm{C}$, and $S_{A u}^{e f f}$ and $S_{C}^{e f f}$ the effective stopping powers in $\mathrm{Au}$ and C. Because $c_{A u} S_{A u}^{e f f} \gg c_{C} S_{C}^{e f f}$ the second term in the denominator can be neglected, resulting in the approximate result. For the example shown in Fig. 6 this approximation is correct within about $20 \%$.

For infinitely large squares the height of the Au spectrum close to the surface is given by

$$
N_{A u} \propto \frac{p_{A u}}{S_{A u}^{e f f}}=\frac{0.5}{S_{A u}^{e f f}}
$$


with $p_{A u}=0.5$ the fraction of the sample area occupied by Au. Comparing the heights of the Au spectra close to the surface from eqs. 1 and 2 the spectra differ by a factor of about two, in agreement with the simulations shown in Fig. 6b). The difference in spectrum height is therefore due to the difference of the effective stopping powers of pure $\mathrm{Au}$ and of $\mathrm{Au}_{0.5} \mathrm{C}_{0.5}$, which differ by a factor of about two. This example illustrates a general problem in the analysis of heterogeneous agglomerate materials: Identical average sample compositions can yield different spectra depending on the lateral arrangement of the materials.

For square sizes above about $50 \mu \mathrm{m}$ all spectra are close to the infinitely large spectrum, while for square sizes below about $0.2 \mu \mathrm{m}$ all spectra are close to the indefinitely small spectrum. For square sizes in between the spectra get complicated due to the finite number of traversed squares, see Fig. 6b).

A spectrum from a laterally inhomogeneous sample can be usually fitted by a one-dimensional depth profile. This is illustrated in Fig. 6c), where the spectrum from the 'Infinitely large' squares sample (dashed line in Fig. 6b)) was fitted by a depth profile (dotted line in Fig. 6b)). The spectra are practically indistinguishable. The depth profile is shown in Fig. 6c) and has only little in common with the real average sample composition. The Au concentration is incorrect by a factor of up to 2.5 , while the $\mathrm{C}$ concentration is incorrect by about $50 \%$. This example shows that the approximation of laterally inhomogeneous samples by one-dimensional depth profiles can yield quantitatively incorrect results. There may be no warning signs to the evaluator, because the spectra can be fitted well. Quantitatively correct analysis of laterally inhomogeneous samples requires taking the correct lateral arrangement of the materials into account. Only for very small sizes of the constituents, i.e. if many constituents are traversed by incident and exit beams, the approximation by atomically mixed slabs is valid.

\section{Conclusions}

The program STRUCTNRA allows the simulation of charged particle energy spectra using RBS, ERDA, NRA, or MEIS from arbitrary two-dimensional distributions of materials. It uses the well-known SIMNRA code as simulation kernel. All SIMNRA features are available for simulations.

For rough thin layers roughness and depth profiles are generally ambiguous. The spectra of rough thin layers often can be modeled by one-dimensional depth profiles. However, these depth profiles have little in common with the real sample structure. Total amounts of elements can be derived with some robustness from count integrals. For moderate roughness, not too large energy 
losses and sufficiently smooth cross-sections count integrals allow to determine total amounts of elements with an uncertainty of the order of less than $10 \%$.

Heterogeneous agglomerate materials (such as sinter materials or paint) are generally ambiguous: Identical average sample compositions can give different spectra for different lateral arrangements of the constituents, and identical spectra can be obtained from different sample compositions: Without additional information about the sample micro-structure it is therefore usually impossible to identify the correct solution. This type of materials therefore cannot be described by depth profiles. Correct simulation of spectra from these materials requires taking the correct two- or three-dimensional sample micro-structure into account.

\section{Acknowledgements}

The silicon grating was generously provided by K. Krieger. The technical assistance with ion beam measurements by J. Dorner and M. Fußeder and scanning electron microscopy investigations by G. Matern are gratefully acknowledged.

\section{References}

[1] J.R. Tesmer and M. Nastasi, Eds. Handbook of Modern Ion Beam Materials Analysis, first ed. Materials Research Society, Pittsburgh, Pennsylvania, 1995.

[2] E. Rauhala, N.P. Barradas, S. Fazinic, M. Mayer, E. Szilágyi, and M. Thompson. Nucl. Instr. Meth. B 244 (2006) 436.

[3] M. Mayer. SIMNRA user's guide. Tech. Rep. IPP 9/113, Max-Planck-Institut für Plasmaphysik, Garching, 1997.

[4] M. Mayer. Nucl. Instr. Meth. B 332 (2014) 176.

[5] C. Jeynes, N.P. Barradas, P.K. Marriott, G. Boudreault, M. Jenkin, E. Wendler, and R.P. Webb. J. Phys. D: Appl. Phys. 36 (2003) R97.

[6] C. Jeynes, M.J. Bailey, N.J. Bright, M.E. Christopher, G.W. Grime, B.N. Jones, V.V. Palitsin, and R.P. Webb. Nucl. Instr. Meth. B 271 (2012) 107.

[7] R.D. Edge and U. Bill. Nucl. Instr. Meth. 168 (1980) 157.

[8] A.R. Knudson. Nucl. Instr. Meth. 168 (1980) 163.

[9] J.R. Bird, P. Duerden, D.D. Cohen, G.B. Smith, and P. Hillery. Nucl. Instr. Meth. 218 (1983) 53. 
[10] C.P. Hobbs, J.W. McMillan, and D.W. Palmer. Nucl. Instr. Meth. B 30 (1988) 342.

[11] M. Wüest and P. Bochsler. Nucl. Instr. Meth. B 71 (1992) 314.

[12] V.S. Shorin and A.N. Sosnin. Nucl. Instr. Meth. B 72 (1992) 452.

[13] H. Metzner, M. Gossla, and Th. Hahn. Nucl. Instr. Meth. B 124 (1997) 567.

[14] I.M. Yesil, W. Assmann, H. Huber, and K.E.G. Löbner. Nucl. Instr. Meth. B 136-138 (1998) 623.

[15] A. Kitamura, T. Tamai, A. Taniike, Y. Furuyama, T. Maeda, N. Ogiwara, and M. Saidoh. Nucl. Instr. Meth. B 134 (1998) 98.

[16] H. Metzner, Th. Hahn, M. Gossla, J. Conrad, and J.-H. Bremer. Nucl. Instr. Meth. B 134 (1998) 249.

[17] T. Sajavaara, K. Arstila, A. Laakso, and J. Keinonen. Nucl. Instr. Meth. B 161-163 (2000) 235.

[18] M. Mayer. Nucl. Instr. Meth. B 194 (2002) 177.

[19] S.L. Molodtsov, A.F. Gurbich, and C. Jeynes. J. Phys. D 41 (2008) 205303.

[20] H. Langhuth, M. Mayer, and S. Lindig. Nucl. Instr. Meth. B 269 (2011) 1811.

[21] M. Mayer, U. von Toussaint, J. Dewalque, O. Dubreuil, C. Henrist, R. Cloots, and F. Mathis. Nucl. Instr. Meth. B 273 (2012) 83.

[22] M.A. Sortica, P.L. Grande, G. Machado, and L. Miotti. J. Appl. Phys. 106 (2009) 114320.

[23] Z. Hajnal, E. Szilágyi, F. Pászti, and G. Battistig. Nucl. Instr. Meth. B 118 (1996) 617.

[24] F. Schiettekatte. Nucl. Instr. Meth. B 266 (2008) 1880.

[25] F. Schiettekatte. Spectrum simulation of rough and nanostructured targets from their 2D and 3D image by Monte Carlo methods. Presented at the 22nd International Conference on Ion Beam Analysis 2015, Opatija, Croatia, 2015.

[26] W. Eckstein and M. Mayer. Nucl. Instr. Meth. B 153 (1999) 337.

[27] A. Gurbich. Nucl. Instr. Meth. B 268 (2010) 1703.

[28] J.F. Ziegler. Nucl. Instr. Meth. B 219-220 (2004) 1027.

[29] W.K. Chu, J.W. Mayer, and M.A. Nicolet. Backscattering Spectrometry. Academic Press, New York, San Francisco, London, 1978.

[30] M. Mayer, W. Eckstein, H. Langhuth, F. Schiettekatte, and U. von Toussaint. Nucl. Instr. Meth. B 269 (2011) 3006. 


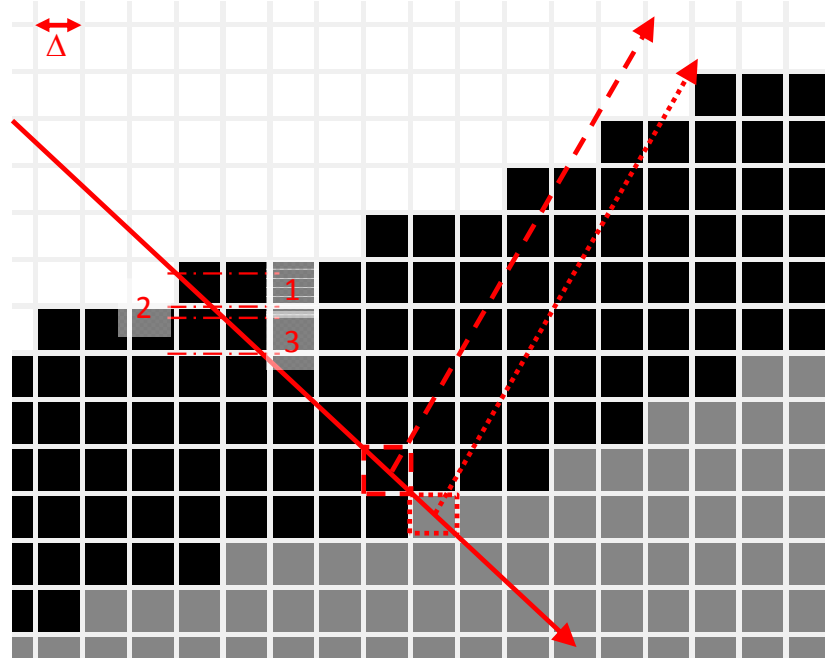

Fig. 1. Schematic representation of the calculational scheme of STRUCTNRA. Different colors indicate different materials. White pixels are assumed to be void, gray and black pixels can contain different materials. $\Delta$ : Pixel size. Solid line: One trajectory of incident particles; Dashed line: Trajectory of outgoing particles from the dashed pixel; Dotted line: Trajectory of outgoing particles from the dotted pixel; Dash-dotted lines: Boundaries of layers 1, 2, and 3 of the target for incident particles $T_{i n}$. 


\section{a)}

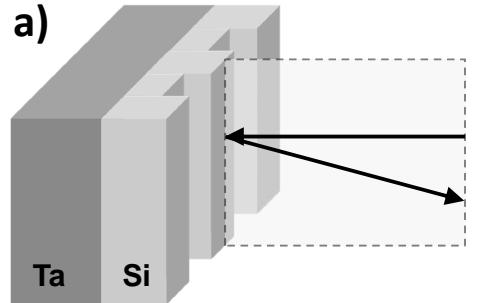

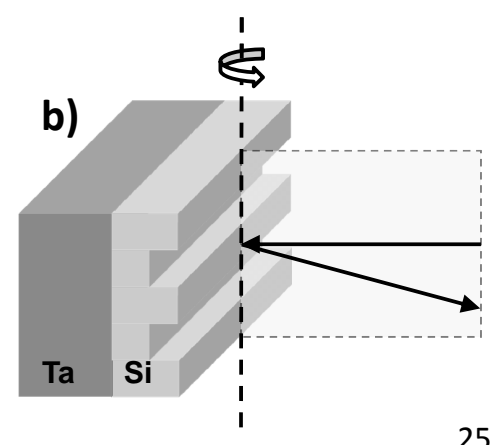

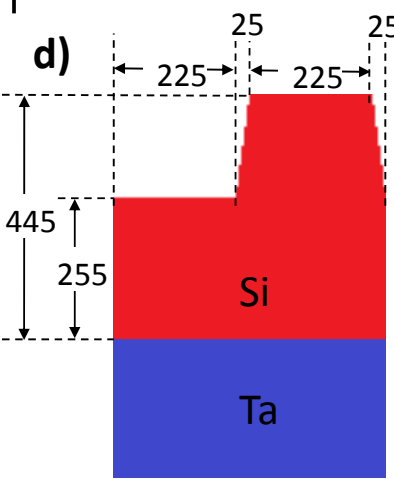

Fig. 2. a) Schematic representation of the scattering geometry in parallel direction with the detection plane (i.e. the plane formed by incident and exit beams) parallel to the silicon grating structure. b) Schematic representation of the scattering geometry in perpendicular direction with the detection plane perpendicular to the silicon grating structure. The thick dashed line is the rotation axis of the sample for measurements at oblique incidence. c) Focused ion beam cross-section trough the silicon grating structure on tantalum. The two arrows indicate the directions of incident and exit beams at a scattering angle of $165^{\circ}$ and normal incidence for the perpendicular geometry. At oblique incidence the plane formed by these two beams is inclined with respect to the paper plane. d) Schematic representation of the target structure used for the simulations shown in Fig. 3. All dimensions are in nm, the pixel size is $5 \mathrm{~nm}$. 


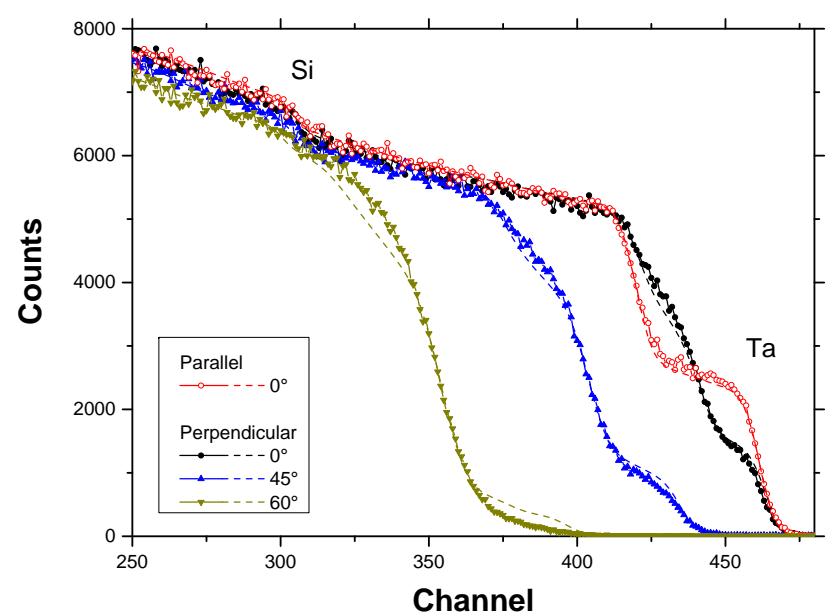

Fig. 3. RBS spectra of the target structure shown in Fig. 2 using $1500 \mathrm{keV}{ }^{4} \mathrm{He}$ ions at a scattering angle of $165^{\circ}$. Symbols: Experimental data; Dashed lines: Simulated data using the target structure shown in Fig. 2d). Red hollow circles: Parallel geometry and normal incidence; Black filled circles: Perpendicular geometry at normal incidence; Blue up triangles: Perpendicular geometry at $45^{\circ}$ angle of incidence; Yellow down triangles: Perpendicular geometry at $60^{\circ}$ angle of incidence. 

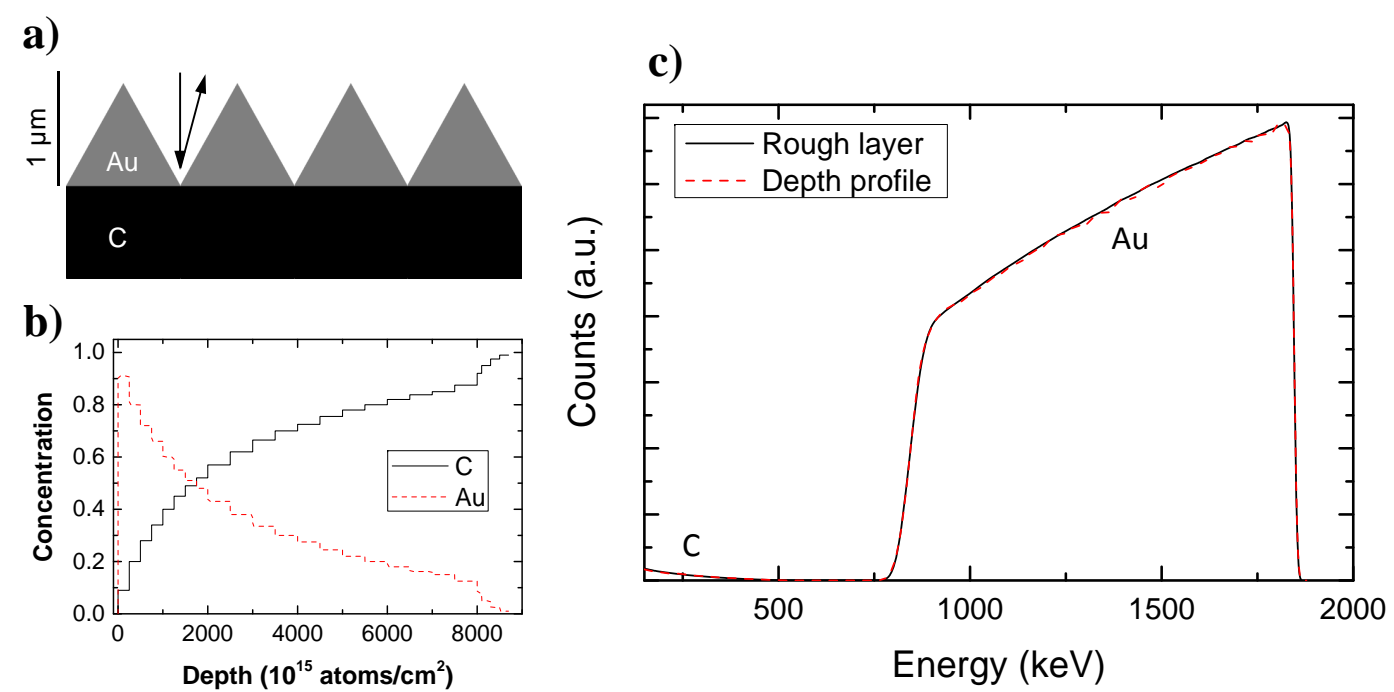

Fig. 4. a) Rough triangular gold layer on top of a graphite substrate. The structure extends infinitely perpendicular to the paper plane. The scattering geometry (normal incidence, scattering angle $165^{\circ}$ ) is indicated by the two arrows. b) Depth profiles of carbon (solid line) and gold (dashed line). c) Simulated back-scattering spectra for $2 \mathrm{MeV}{ }^{4} \mathrm{He}$, Solid line: Spectrum for the rough gold layer from a); Dashed line: Spectrum for the gold/carbon depth profiles from b). 

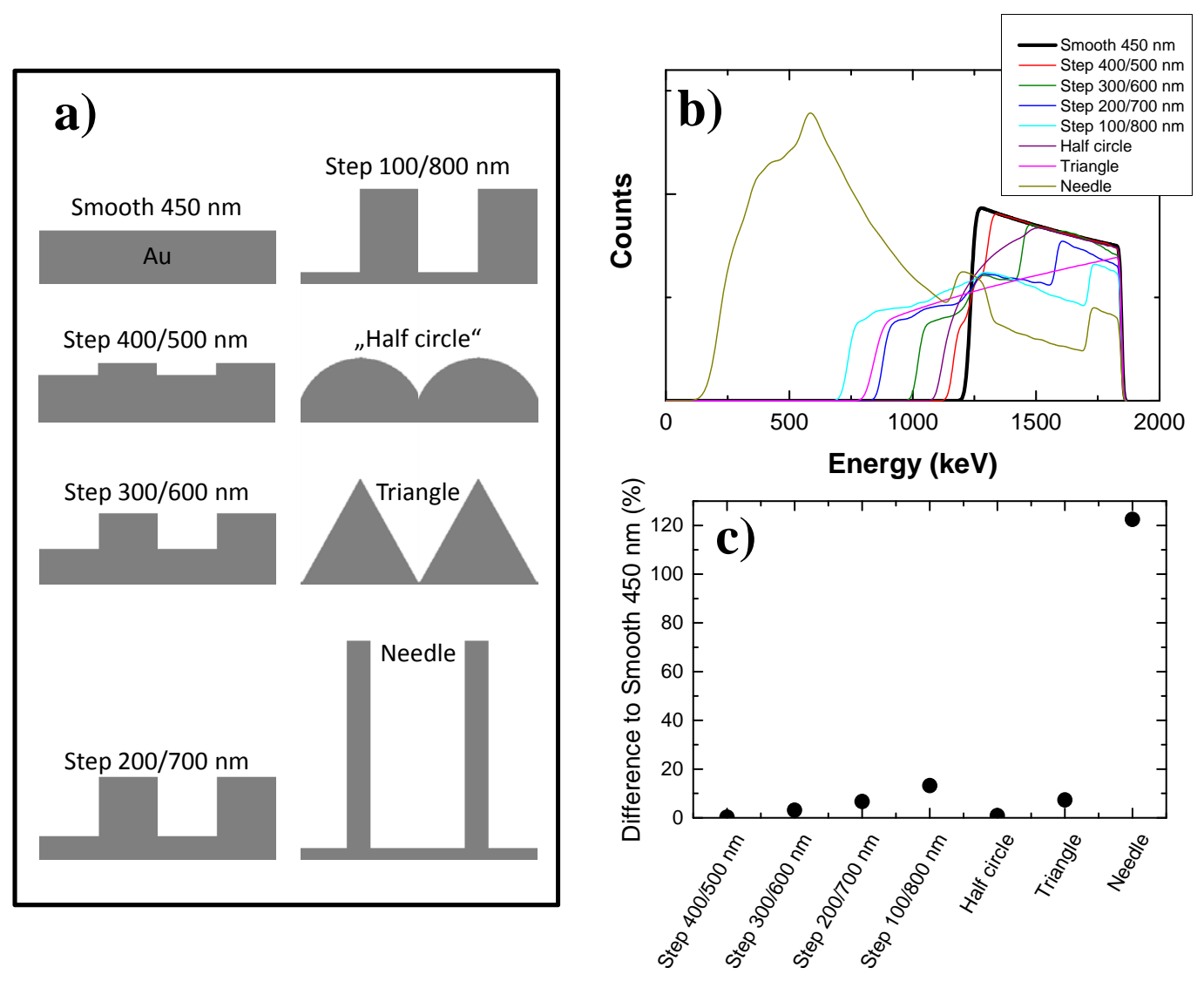

Fig. 5. a) Smooth and rough gold layers with different layer thickness distributions. The smooth layer has a thickness of $450 \mathrm{~nm}$, the two thicknesses of the 'Step' distributions are indicated. The total amount of gold is $2.66 \times 10^{18}$ atoms $/ \mathrm{cm}^{2}$ in all cases. The structures extend infinitely perpendicular to the paper plane and have periodic boundary conditions in horizontal direction. b) Simulated RBS spectra of the gold layers from a) for $2 \mathrm{MeV}^{4} \mathrm{He}$ ions, normal incidence, scattering angle $165^{\circ}$. c) Difference of the count integrals of the spectra in b) to the count integral of the smooth layer. 

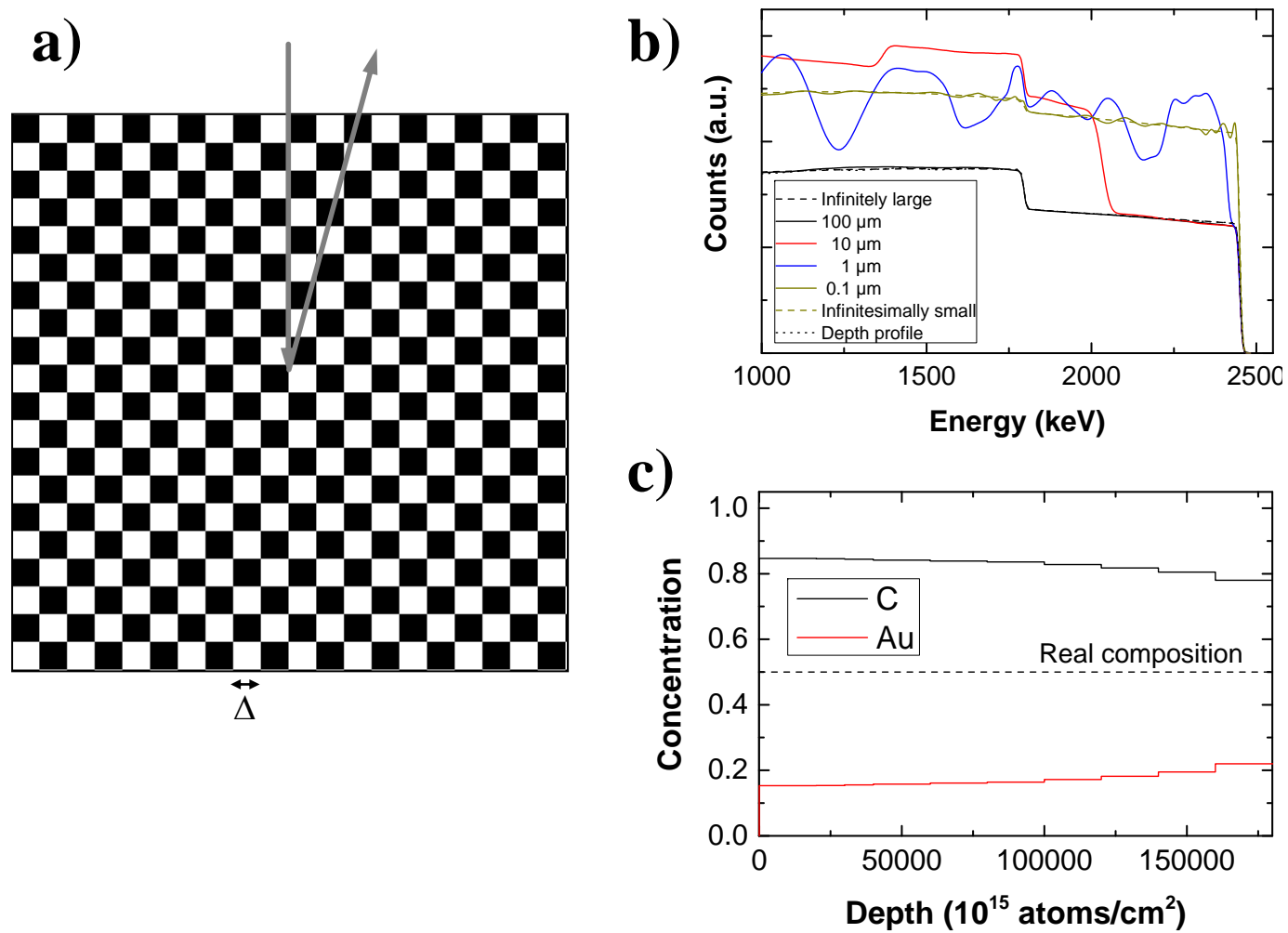

Fig. 6. a) Chessboard-like arrangement of carbon (black) and gold (white) with square size $\Delta$. The structure extends infinitely perpendicular to the paper plane. The scattering geometry with normal incidence and a scattering angle of $165^{\circ}$ is indicated by the arrows. b) Calculated back-scattering spectra for $2.5 \mathrm{MeV}$ protons, normal incidence, $165^{\circ}$ scattering angle for different square sizes. Solid lines: Calculations by STRUCTNRA for square sizes from 0.1 to $100 \mu \mathrm{m}$; Dashed lines: Calculations by SIMNRA for infinitely large and indefinitely small squares; Dotted line: Calculated spectrum for a one-dimensional material distribution and the depth profile shown in c) (the dotted line is hardly visible due to overlap with the dashed line for 'Infinitely large')). c) Depth profiles of $\mathrm{Au}$ and $\mathrm{C}$ used for the calculation of the dotted line in b). 